UDK 78(438 Varšava)"17"

Alina Żórawska-Witkowska

Institut für Musikwissenschaft, Universität Warschau

Muzikološki inštitut Univerze v Varšavi

\title{
Warschau und seine musikalische Identität in der ersten Hälfte des 18. Jahrhundert (die so genannten Sachsenzeiten)
}

\section{Varšava in njena glasbena identiteta $\mathrm{v}$ prvi polovici 18. stoletja (tako imenovano saško obdobje)}

\section{ZUSAMMENFASSUNG}

An Hand der Anzahl von Publikationen in den letzten 50 Jahren über die geschichtliche Gemeinschaft der Länder und Völker in Mitteleuropa oder - wie es die Forscher der Geschichte der Republik Beider Nationen mögen - in Mittelosteuropa, ist das eine außerordentlich frappierende Thematik für Historiker verschiedener Fachgebiete und jüngst sogar modern. Davon, dass die Sprache der Musik eins der universellsten Motive allgemeiner europäischer Tradition ist, zeugen die in gewissen Epochen dominierenden Ideen, Stile und Gattungen, die zu einem universellen Gut für den gesamten Kontinent geworden sind. Existierte aber eine musikalische Identität, die nur für Mitteleuropa spezifisch gewesen ist? Vielleicht geben die Ergebnisse unserer Konferenz darauf eine Antwort. Die Autorin begrenzte ihre Betrachtungen auf eine Stadt, auf Warschau, und auf den Zeitraum der Herrschaft zweier sächsischer Könige der Wettin-Dynastie in Polen - August II. (1697-1733) und Augusta III. (1734-1764). Diese beiden Monarchen waren gleichzeitig sächsische Kurfürsten und verbrachten mehr Zeit im heimatli-
POVZETEK

Glede na veliko število publikacij o skupni zgodovini dežel in narodov Srednje Evrope ali - kot imajo radi raziskovalci državne zgodovine obeh narodov - srednjevzhodne Evrope v zadnjih 50. letih gre za izjemno osupljivo in $\mathrm{v}$ zadnjem času celo moderno temo zgodovinarjev različnih strok. Na to, da je glasbeni jezik eden najbolj univerzalnih motivov skupne evropske tradicije, kažejo v določenih obdobjih prevladujoče ideje, stili in zvrsti, ki so postali univerzalna dobrina za celotni kontinent. Je torej obstajala glasbena identiteta, ki je bila značilna le za Srednjo Evropo? Morda bodo rezultati naše konference podali odgovor na to vprašanje.

Avtorica je svoja opazovanja omejila na Varšavo v obdobju vladavine dveh saških kraljev dinastije Wettiner na Poljskem - Avgusta II. (1697-1733) in Avgusta III. (1734-1764). Oba omenjena monarha, ki sta bila istočasno saška volilna kneza, sta preživela več časa v domačem Dresdnu kakor v Varšavi. Skupna značilnost njunega poljskega mecenstva je bila tesna povezanost med Varšavo in Dresdnom, od koder so na Poljsko prišli številni umetniki saškega dvora - pevci, plesalci, igralci, skladatelji in izbrani glasbeni virtuozi. Tako se je repertoar 
MUZIKOLOŠKI ZBORNIK • MUSICOLOGICAL ANNUAL XL

chen Dresden als in Warschau. Der gemeinsame Charakter ihres polnischen Mäzenatentums bestand in der engen Verbindung zwischen Warschau und Dresden, aus dem zahlreiche Künstler des sächsischen Hofes - Sänger, Tänzer, Schauspieler, Komponisten und ausgewählte Musikvirtuosen nach Polen kamen. Das brachte eine Übertragung des zuerst in Dresden präsentierten Repertoires auf Warschauer Boden mit sich. Man muss zugestehen, dass es ein ausgezeichnetes und vielseitiges Repertoire war, nicht nur aus Frankreich und Italien importiert oder exemploarisch an dem Schaffen dieser Länder orientiert, sondern auch ein originales, von deutschen und tschechischen Komponisten des polnisch-sächsischen Hofes geschaffen.

Warschau besaß aber auch Initiative gegenüber Dresden, nämlich die eigenständig und direkt von Paris nach Polen geholten Tragédie en musique J.-B. Lully (1700-1703), Ballet d'action (schon 20er Jahre), von Venedig - Intermezzi (1716-1718) oder auch die aus der örtlichen Tradition hervorgegangenen Janitscharen-Kapelle. Damit wurden also im Laufe der besprochenen 60 Jahre französische und italienische Opern, französische, italienische und deutsche Komödien, italienische Oratorien, deutsche instrumentale und gewiss auch kirchliche Musik aufgeführt; eine enorme Bedeutung erreichten die polnische Tänze - Polonaise und Mazurka. Eine Reihe von Werken, zum Beispiel Gelegenheitsserenaden, entstanden in Warschau und für Warschau. Und trotz aller Pracht der Warschauer Musikkultur in dieser Epoche muss deutlich hervorgehoben werden, dass Warschau im Bereich der professionellen Schöpfungen fast ausnahmslos die nehmende Seite gewesen war.

Erst die Herrschaft des Landsmannes Stanisław August Poniatowski (1764-95) trug zur Wiedergeburt der lokalen kompositorischen Umwelt bei und zum Entstehen ung ersten Nationalopern, die eine sehr große Rolle in den Theatern durch das ganze Polen spielten und die Richtung wiesen, für die Entwicklung der polnischen Oper im 19. Jahrhundert. izveden v Dresdnu prenesel na varšavska tla. Treba je priznati, da je bil to odličen in vsestranski repertoar, ki ni bil le uvožen iz Francije in Italije ali eksemplarično orientiran na ustvarjanje $\mathrm{v}$ ome= njenih deželah, temveč tudi izviren repertoar, ki so ga ustvarili nemški in češki skladatelji poljskosaškega dvora.

Varšava je sčasoma Dresdnu prevzela repertoarno iniciavito, saj sta bili neposredno iz Pariza na Poljsko prinešeni Tragédie en musique J.-B. Lullyja (17001703) in Ballet d'action (že v 20. letih), iz Benetk Intermezzi (1716-1718), iz lokalne tradicije pa je izšla janičarska kapela. Tako so bile torej v 60 . letih izvedene francoske in italijanske opere, francoske, italijanske ter nemške komedije, italijanski oratoriji, nemška instrumentalna in seveda tudi cerkvena glasba; velikanski pomen pa sta dosegla poljska plesa - poloneza in mazurka. Vrsta del, na primer priložnostne serenade, je nastala $v$ Varšavi in za Varšavo. Kljub vsemu razkošju varšavske glasbene kulture v tem obdobju pa je potrebno posebej poudariti, da je bila Varšava na področju skladateljskega ustvarjanja docela nesamostojna.

Šele med vladavino rojaka Stanislawa Avgusta Poniatowskega (1764-1795) je prišlo do ponovnega rojstva lokalnega skladateljskega okolja in nastanka prve nacionalne opere. Slednja je imela posebej pomembno vlogo v gledališčih po vsej Poljski in je nakazala smer za razvoj poljske opere v 19. stoletju. 
MUZIKOLOŠKI ZBORNIK • MUSICOLOGICAL ANNUAL XL

An Hand der Anzahl von Publikationen über die geschichtliche Gemeinschaft der Länder und Völker Europas - allgemein gesagt der in Mitteleuropa -, die in den letzten 50 Jahren diesem Thema gewidmet wurden, darf man annehmen, dass dies eine außerordentlich frappierende Thematik für den Historiker geworden und jüngst sogar in Mode gekommen ist. »In der heutigen Historiografie werden die Vielseitigkeit der Auffassungen und eine vergleichende, breite Betrachtungsweise über die Grenzen hinaus zu einem fundamentalen Postulat, unabdingbar für das Studium jeder menschlichen Gemeinschaft irgendeiner Gesamtheit, einschließlich der staatlichen, nationalen oder religiösen « ${ }^{1}$.

Diskutiert werden aber muss das Problem der Art und Weise der Unterscheidung jener Gemeinschaften, denn hinter den verschiedenen Vorschlägen stehen am häufigsten abweichende Erfahrungen sowohl die, dem jeweiligen Historiker eigen sind als auch die seines Volkes.

Ich habe seinerzeit - als Staatsbürgerin eines Landes, das fast vollständig von der Union der Sozialistischen Sowjetrepubliken abhängig war - aus eigener Betrachtung gewusst, dass sich Europa einzig und allein in West - sieggekrönt, frei, schön, reich und für mich unerreichbar - sowie in Ost teilt - getrennt durch den Eisernen Vorhang, abhängig vom kommunistischen Sowjetimperium - arm, unterdrückt, eingesperrt und bar jeder Hoffnung auf eine Änderung. Jene dichotomische Unterteilung war jedoch nicht nur das subjektive Empfinden der Menschen, die in einem der Länder wohnten, die ja seinerzeit euphemistisch Volksdemokratien genannt worden waren. Viele Wissenschaftler sahen und sehen Europa auch heute noch so, unter ihnen ebenfalls der britische Historiker Norman Davies, der in seinen hervorragenden Werken über Polen ${ }^{2}$ sowie in der gleichfalls faszinierenden Geschichte Europas ${ }^{3}$ unseren Kontinent hauptsächlich in Ost und West teilt und gemäß den verschiedenen Demarkationslinien (geografische, religiöse, politische, wirtschaftliche etc.) das heutige Polen entweder zum Westen oder auch zu Osteuropa zählt, manchmal situiert er es auch ins »Herz von Europa « ${ }^{4}$. Davies hebt dabei hervor, dass jene bipolare Aufteilung »die starke und tief greifende historische Teilung zwischen dem Norden und dem Süden völlig ignoriert" und dass "gewiss jeder verantwortungsbewusste Historiker oder Geograf zu der Überzeugung käme, Europa dürfe nicht in zwei Teile geteilt werden, sondern mindestens in fünf oder sechs ${ }^{5}$.

Wenn wir nun die Diskussion zum Thema des in unserer Konferenz verankerten Problems aufnehmen, dann sollten wir immerhin eine möglichst genaue

1 Jerzy Kłoczowski, Vorwort zur Historia Europy Środkowo-Wschodniej [Geschichte Mittel-Ost-Europas], hrsg. von Jerzy Kłoczowski, Instytut Europy Środkowo Wschodniej, Lublin 2000, t.I, s.6.

2 Norman Davies, God's Playground. A History of Poland, vol. I The Origins to 1795, Columbia University Press, New York 1982, Polnische Ausgabe Boże igrzysko. Historia Polski, t. I Od początków do roku 1795, autorisierte Übersetzung von Elżbieta Tabakowska, Wydawnictwo Znak, Kraków 1990, S. 53-54 u. 62-63.

3 Norman Davies, Europe. A History, Oxford University Press 1996, Polnische Ausgabe Europa. Rozprawa historyka $z$ historiq, Übers. Elżbieta Tabakowska, Wydawnictwo Znak, Kraków 2002.

4 N. Davies, Europa..., S. 43, Karte Nr. 3. Ost-West: Teilungslinie Europas.

5 ebenda, S. 54. 
Vorstellung vom Terminus »Mitteleuropa" haben. Aber die Sache ist die, dass jener Begriff überaus unscharf ist und sowohl in der Vergangenheit als auch in der heutigen Zeit unterschiedlich verstanden wird, darüber hinaus politisch auch stark akzentuiert ist. Die am Ende des 19. Jahrhunderts entstandene Konzeption eines »Mitteleuropas « umfasste ein Gebiet, das geprägt war von der Sphäre politischer und kultureller deutscher Einflüsse. In der Zwischenkriegszeit entstand der etwas modifizierte Begriff von einem »Mittelosteuropa«, der im Zusammenhang mit dem Erscheinen von Staaten auf der politischen Landkarte des Kontinents geprägt worden war, die ihre Souveränität neu erhalten oder wiedererlangt hatten: Finnland, Polen, die Tschechoslowakei sowie Jugoslawien. Mitte des 20. Jahrhunderts schlug der herausragende polnische Historiker Oskar Halecki vor, neben dem romanisch-germanischen Westen und dem slawischen Osten, auch das breit verstandene Mitteleuropa zu separieren mit zwei deutlichen Gliedern: dem deutschsprachigen Mittel-West-Europa sowie dem zwischen Deutschland und Russland liegenden Mittel-Ost-Europa ${ }^{6}$. Heute lancieren polnische Historiker, die einst die Richtungen der Expansion ihres Landes vertieften, gerade eben diesen Begriff: Mittel-Ost-Europa. Damit umfassen sie jene »europäischen Gebiete, die über eine Reihe von Jahrhunderten zur Republik Beider Nationen, das heißt zum Königreich Polen und dem Großfürstentum Litauen sowie zu den historischen Königreichen Tschechien und Ungarn gehörten " ${ }^{7}$. Das sind gegenwärtig solche Staaten wie Polen, Litauen, Weißrussland, die Ukraine, Tschechien, die Slowakei, Ungarn, Kroatien und ein bedeutender Teil von Rumänien (Siebenbürgen), dazu kommen dann auch noch der russische Bezirk Königsberg, Lettland und Estland.

Ivan Klemenčič, Ideengeber und Organisator unserer Konferenz, hat eine eigene, im Übrigen auch eine sehr »slowenische« Probe von einem geografischen Umriss und der historischen Grenzen »Mitteleuropas« vorgelegt. Aus seinem uns empfohlenen Essay ${ }^{8}$ geht hervor, dass er unter diesem Begriff diejenigen kleinen Länder und Völker versteht, die im Verlauf von über 1000 Jahren zum Imperium der Habsburger gehörten, das sind: Kroatien, Slowenien, Tschechien, Mähren, die Slowakei, Ungarn und Norditalien. Mit einem gewissen Zögern ist er bereit, auch Polen in diese posthabsburgische Familie mit aufzunehmen. Obgleich es in der Mitte des Kontinents liegt und seinerzeit opferbereit die Rolle einer Vormauer des Christentums (des römischen) ausfüllte, so überragt es jedoch bedeutend die mitteleuropäischen Nachbarn durch die Größe seines Territoriums und seiner Population; darüber hinaus gehörte nur ein geringer Teil seines Gebiets - und dies für relativ kurze Zeit - zum Imperium der Habsburger

6 Oskar Halecki, Limits and Divisions of European History, ... London 1950, deutsche Ausgabe Europa. Grenzen und Gliederung seiner Geschichte,... Darmstadt 1957, polnische Ausgabe Historia Europy-jej granice i podzia $3 y$, Instytut Europy CErodkowej, Lublin 1994.

7 Historia Europy Srodkowo-Wschodniej ..., S. 7.

8 Ivan Klemenèiè, Music history beyond borders and the borders of the musical Central Europe, www. oeaw.ac.at/ mufo/agora/klemencic.html. 
MUZIKOLOŠKI ZBORNIK • MUSICOLOGICAL ANNUAL XL

(hierbei handelt es sich um Schlesien sowie um die von Österreich besetzten Gebiete, also um das so genannte Galizien und Lodomerien). Aber überzeugt ist Klemenčič vor allem davon - hier folgt er zweifellos der literarischen Vorstellung von Milan Kundera - dass jener mitteleuropäische Raum, den die kleinen Völker einnehmen, die einst so einträchtig und mit Hochachtung vor der nationalen Autonomie in der gemeinsamen Monarchie der Habsburger gelebt hatten, sich noch heute durch eine fühlbare »außergewöhnliche Kondensation des Geistes" sowie durch eine kulturelle Gemeinschaft auszeichnet. Somit soll der Anruf jenes Geistes wohl auch das Ziel unserer Konferenz sein.

Davon, dass die Sprache der Musik eins der universellsten Motive der allgemeinen europäischen Tradition darstellt, zeugen die dominierenden Ideen in gewissen Epochen, die Stile und Gattungen, die für den gesamten Kontinent zu einem universellen Gut geworden waren (zum Beispiel im Mittelalter der gregorianische Choral, im Barock das italienische Dramma per musica und im Klassizismus die Sonatenform). Existierte jedoch eine musikalische Identität, spezifisch nur für Mitteleuropa? Nun, wir werden sehen.

Meine Überlegungen begrenzen sich auf nur eine Stadt und auf 60 Jahre eines Jahrhunderts. Jedoch erscheint mir sowohl die Stadt als auch das Jahrhundert aus der Sicht des Forschers sehr interessant. Warschau, beinahe in der Mitte Europas liegend, war im 18. Jahrhundert das Domizil der letzten drei polnischen Könige sowie die Hauptstadt der in seiner einstigen Macht niedergehenden Republik Beider Nationen, des Unionsstaates (im Jahre 1569 gebildet), und dieser Staat vereinte unter einer Krone sowohl das Königreich Polen als auch das Großfürstentum Litauen und war darüber hinaus die ersten 63 Jahre in diesem Jahrhundert gleichfalls noch in Personalunion mit Sachsen verbunden. Die faktischen Staatsgrenzen reichten damals im Norden - bis an die Ostsee, im Süden - an Österreich und an das Osmanische Imperium, im Westen - an Brandenburg und Schlesien, im Osten - an das russische Imperium. Es ist hierbei also unverkennbar zu sehen, dass die Republik Beider Nationen geografisch mehr in Richtung Ost-Europa tendierte, jedoch die religiösen, politischen und kulturellen Bindungen sowie die Ambitionen der Staatsbürger seit Jahrhunderten auf West-Europa gerichtet waren. Die Republik Beider Nationen war ein Vielvölkerstaat und damit gleichzeitig auch kulturell und religiös mannigfaltig. Bis zur ersten Teilung des Landes, die im Jahre 1772 erfolgte, repräsentierte Polen eine Fläche von $733500 \mathrm{~km}^{2}$ und war hinsichtlich seiner Größe - nach Russland und Frankreich - der drittgrößte europäische Staat, der im mittel-östlichen Teil des Kontinents kulturell dominierte. Nicht ganz ein Vierteljahrhundert später, als Folge der dritten Teilung des Staates zwischen den drei Mächten - Preußen, Russland und Österreich - verschwand der polnische Staat von der politischen Landkarte des Kontinents. Trotz dieser heraufziehenden politischen Katastrophe brachte das 18. Jahrhundert aber auch eine Reihe erfolgreicher kultureller Versuche der Wiedergeburt und Modernisierung des Landes, was dem Volk dazu diente, die langen Jahre der Knechtschaft zu überdauern. 
MUZIKOLOŠKI ZBORNIK - MUSICOLOGICAL ANNUAL XL

Am Ende des 17. Jh. unterschied sich Warschau in vielerlei Hinsicht von anderen europäischen Hauptstädten. Zwar zählte die Stadt rund 20000 Einwohner, also mehr oder weniger so viel wie die damaligen Städte Dresden und Berlin, aber die Gruppe der Bürger war die kleinste und auch die schwächste. Auch der Königshof von Johann III. Sobieski, der große Sieger über die Türken, spielte nur eine kleine Rolle. Er bevorzuge es, in seinen privaten Residenzen in Kleinrussland oder in der Warschauer Vorstadt Wilanów zu verweilen. $\mathrm{Zu}$ dieser Zeit gaben die Magnaten sowie die Geistlichkeit den Ton in Warschau an und der kulturelle Stand der Stadt - sowohl der künstlerische als auch der geistige - präsentierte sich eher erbärmlich.

Diese Situation änderte sich im 18. Jh. grundsätzlich, obwohl sich diese Evolution nicht ohne Schwierigkeiten vollzog. August II., seit 1697 der neue Herrscher über die Republik Beider Nationen und gleichzeitig Kurfürst von Sachsen, hatte gegenüber Warschau große Pläne und als Mäzen verband er mit dieser Stadt anfangs all seine Ambitionen. Jedoch erwiesen sich bereits die ersten Jahre seiner Herrschaft überaus glücklos. Der Krieg im Norden, die zeitweilige Entthronung dieses Herrschers (1706-1709) und eine Reihe von Naturkatastrophen (1708-15), in deren Ergebnis die Einwohnerzahl von Warschau unter die 10000 fiel, erzwangen die Notwendigkeit, die Absichten der komplizierten und schwierigen Wirklichkeit anzupassen. Erst im Jahre 1716 begann ein fast 80 Jahre dauernder Zeitraum des Friedens, der Stabilisierung und der schnellen Entwicklung der Stadt, die in demografischer Hinsicht einen über $10 \mathrm{fa}$ chen großen Zuwachs erreichte: 1754 - 24000 Einwohner, 1764 - 30 000, 1784 - 63000 und im Jahre 1792 - über 110 000. Die Anwesenheit des königlichen Hofes, selbst dann - wie es bei beiden sächsischen Königen der Fall war -, wenn dies nur periodischen Charakter hatte, bewirkte einen zahlreichen, laufenden Zustrom von Ausländern (Deutsche, Franzosen, Italiener, Tschechen) und bereicherte - der Natur der Sache nach - das multinationale Antlitz der Stadt (Polen, Litauer, Rutener, Kleinrussen, Armenier, Juden usw.). Warschau war aus diesem Grund ein höchst kosmopolitisches Zentrum sowohl für den Kontinent als auch für den Rest des Landes, wo weiterhin noch die barockesarmatische Kultur des Adels aus dem 17. Jh. dominierte, die eine spezifische Symbiose der westlichen und östlichen Kultur darstellte, zudem aber verbunden war mit einem übermäßigen Hang nach bürgerlichen Freiheiten, mit Xenophobie und standesdünkelnder Megalomanie.

Die so genannte Sachsenzeit umfasst die Jahre der Herrschaft von August II. (1697-1733, mit der Unterbrechung 1706-1709 durch Stanisław Leszczyński) sowie August III. (1734-1763), also zweier Deutscher aus der sächsischen Wettin-Dynastie. Als ihr Nachfolger bestieg der Pole Stanisław August Poniatowski (1764-1795) den polnischen Thron. Seit dem Augenblick, als Warschau Polens Hauptstadt geworden war (1596) bis zum Verlust der Souveränität des Landes (1795), bestimmte gewöhnlich der Königshof den musikalischen Ton der Stadt, die übrigen Sphären spielten eine untergeordnete Rolle.

Wenngleich nun beide Könige einen unterschiedlichen musikalischen Ge- 
schmack besaßen, so zeichnete ihr Mäzenatentum eine enge Verbindung Warschaus mit Dresden aus, sowohl in Bezug auf die Organisation des künstlerischen Personals als auch in Hinsicht auf das Repertoire. Das heimatliche Dresden, im Übrigen eins der hervorstechendsten Zentren für Musik und Theater in der zeitgenössischen Welt, bildete für August II. (vielleicht in geringerem Maße) und für August III. (in höherem Maße) den künstlerischen Mittelpunkt und das Fundament ihrer Warschauer Residenzen.

Aus Dresden nahmen sie zahlreiche Sänger, Tänzer, Schauspieler und ausgewählte Musiker nach Warschau mit, ja selbst das technische Personal für das Theater. Daher bewirkte jede Rückkehr des Hofes nach Sachsen auch eine enorme Schwächung des pulsierenden Lebens in Warschau und eine Stagnation der kulturellen Unternehmungen. Die Anwesenheit dieser Monarchen bestimmte den Rhythmus und die Intensivität des künstlerischen Lebens der Stadt und machte sie damit nur periodisch zu einem musikalischen und Theaterzentrum in diesem Teil Europas. Erst der durch den Ausbruch des 7jährigen Krieges erzwungene 7 jährige Aufenthalt Augusts III. in Warschau trug zu einer ständigen herausragenden kulturellen Entwicklung bei, selbst um den Preis, dass diese Entfaltung nicht der Sorge des Mäzens um seine polnisch-litauischen Untertanen entsprang, sondern der Realisierung eines eigenen ästhetischen Bedürfnisses. Gerade in dieser Zeit (nach über 100 Jahren) gewann Warschau den Glanz einer tatsächlichen königlichen Residenz wieder zurück und das Leben der Oper näherte sich dem Dresdner Niveau vor dem Kriegsausbruch.

Wie ich bereits ausgeführt habe, hatte August II. in der ersten Phase seiner königlichen Herrschaft (1697-1704) all seine Pläne als Mäzen mit Warschau verbunden und nicht mit Dresden?'. In Warschau residierte seine ca. 40 Personen umfassende Königlich Pohlnische Capelle, die sich aus ehemaligen Musikern Johann III. Sobieskis sowie des Sachsenhofes zusammensetzte, mit zwei Kapellmeistern an der Spitze - dem Deutschen Johann Christoph Schmidt und dem Polen Jacek Różycki. Die nationale Besetzung dieses Ensembles war höchst verschieden. Es wurde nämlich von Italienern, Polen, Deutschen und Franzosen gebildet. In Warschau wurden auch verschiedene Theatertruppen angesiedelt, die für die Bedürfnisse dieser Stadt engagiert worden waren: ein Ensemble der italienischen Komödie unter der Direktion von Gennaro Sacco (Februar - Mai 1699), Ensembles der französischen Komödie unter Denis Nanteuil (Mai - August 1699?) und Jean de Fonpré (Mai 1700-1703/04?), sowie

\footnotetext{
9 Näheres über die Musik am Hofe August II. in: Alina Żórawska-Witkowska, Muzyka na dworze Augusta II w Warszawie [Die Musik am Hofe August II. in Warschau], Arx Regia, Warszawa 1997. Siehe auch: A.ŻórawskaWitkowska, Il teatro musicale francese alla corte di Augusto II a Varsavia, w: Actes du Congrčs International Théâtre, Musique et Arts dans les Cours Européennes de la Renaisance et du Baroque. Varsovie, 23-28 septembre 1996, Red. Kazimierz Sabik, Éditions de l'Université de Varsovie, Varsovie 1997, S. 425-445; A.ŻórawskaWitkowska, Il teatro musicale italiano a Varsavia al tempo di Augusto II il Forte (1697-173). Un tentativo di ricostruzione del repertorio, w: Il teatro musicale italiano nel Sacro Romano Impero nei secoli XVII e XVIII. Atti del VII Convegno internazionale sulla musica italiana nei secolo XVII-XVIII Loveno di Menaggio (Como), 15-17 luglio 1997, a cura di Alberto Colzani, Norbert Dubowy, Andrea Luppi, Maurizio Padoan, A.M.I.S., Como 1999, S.293-309.
} 
auch ein ca. 50 Personen umfassendes Ensemble der französischen Oper unter der Leitung von Louis Deseschaliers (Juni 1700 - 1703/04?).

Die zweite Phase des Warschauer Mäzenatentums von August II. umfasst die Jahre 1716 - 1733, als die Enttäuschung dieses Königs über Polen sowie der Polen über diesen König auf beiden Seiten dazu führte, alle Absichten der komplizierten und schweren Wirklichkeit anzupassen. Das Hauptorchester des polnischen Hofes war damals die aus mehr als zehn Musiker (12-15 Personen) gebildete "pohlnische Capelle», die aus der polnischen Kasse bezahlt wurde, sich jedoch ausschließlich aus Künstlern des Deutschen Reiches zusammensetzte. In dieser Kapelle hatten unter anderem solche Musiker wie Johann Joachim Quantz, František Benda und Jiři Čart ihre großartige Karriere begonnen. Allein die Stellung des Organisten - unabhängig von der Kapelle - wurde dem Polen Piotr Kosmowski übertragen. Konzertmeister des Ensembles war Heinrich Schulze und die Funktion des Kapellmeisters teilten sich Giovanni Alberto Ristori und Louis André, in Abhängigkeit davon, welche der zwei in Dresden residierenden Theatertruppen im gegebenen Moment nach Warschau verlegt wurde - die Truppe der italienischen Komödie unter der Leitung von Tommaso Ristori (in Warschau: 1716-18, 1725-29, 1730) oder das königliche Ensemble der französischen Komödie und des Balletts (in Warschau: 1715, 1720-24, 1725-26).

Ein anderes und überaus originelles Ensemble, das vom polnischen Hof bezahlt wurde, war die Janitscharen-Kapelle. Ihre instrumentale Besetzung ahmte türkische Ensembles getreu nach, sie wurde jedoch aus 24 Musikern gebildet, die aus dem Gebiet der Republik Beider Nationen kamen (polnisch lautende Namen). Diese Art von Kapelle, in Polen und Litauen seit dem 17. Jh. existent, wurde von den Forschern als eine der Erscheinungen des Sarmatismus (einer Mode nach Orientalismus) anerkannt. Für August II. wurde jene Janitscharen-Kapelle zu einem Symbol für die Stärke des polnischen Königs, dessen Herrschaftsgebiet bis an das türkische Imperium reichte. Wohl auch deshalb beorderte August II. jenes Ensemble anlässlich der für ihn politisch wichtigen Ereignisse nach Sachsen, wie zum Beispiel bei den berühmten Hochzeitsfeierlichkeiten zu Ehren von Friedrich August und der Erzherzogin Maria Josepha im Jahre 1719 oder für das bekannte Kampament von 1730 in Radewitz, das für den Preußenkönig Friedrich Wilhelm I. und dessen Sohn Friedrich organisiert worden war.

Die Aufenthalte von August II. sowie des Vaters August III. waren auch von einer Gruppe Hof-Trompeter und Pauker sowie Bock-Pfeiffer begleitet worden, die von den entsprechenden Ensembles des Dresdner Hofes ausgewählt wurden, einer Gruppe also, die sich aus Deutschen oder Tschechen zusammensetzte.

Forschungen haben bewiesen, dass Polen eins der ersten Länder war, das auf seinem Boden die Werke Moličres ${ }^{10}$ einführte. Und alles weißt darauf hin,

\footnotetext{
${ }^{10}$ Karolina Targosz, Dwór królowej Marysieńki Sobieskiej ogniskiem recepcji teatru francuskiego [Der Hof von Marysienka Sobieska als Hort der Rezeption des französischen Theaters], „Barok. Historia-Literatura-Sztuka“ II/1, 1995, S.43-83, passim.
} 
dass man Warschau ebenso in Bezug auf das Opernschaffen von Jean-Baptiste Lully einreihen darf in den exklusiven Kreis französischer Zentren außerhalb des Mutterlandes, die damals Werke dieses Meisters aufführten (Amsterdam, Antwerpen, Brüssel, Gent, Den Haag, London, Ansbach, Bonn, Hamburg, Wolfenbüttel), darunter bestimmt seine Tragédies en musique wie Atys, Armide und Thésée. Das grand sičcle Ludwig XIV. bildete für August II. nur in den ersten Jahren seiner Herrschaft über die Republik Beider Nationen eine Stütze. Später führte er, der von Paris diktierten Mode nacheifernd, in seinen Theatern den Geschmack der Regentschaft ein und präferierte leichtere Gattungen wie Comédie-ballets, Comédie lyriques, Komödien mit Divertissements, Fragments des ballets und darüber hinaus die in der Geschichte ersten eigenständigen Ballette, das als Urmuster anerkannte Ballet d'action, 1724 Le Ballet d'aprés Horace mit der Musik von Jean-Joseph Mouret, 1725/26 Les Caractères de la danse mit der Musik von Jean-Ferry Rebel. Im Bereich des französischen dramatischen Repertoires ist deutlich die Neigung von August II. zur bewährten Klassik aus der zweiten Hälfte des 17. Jh. sowie aus dem Beginn des 18. Jh. zu beobachten. In Warschau und in Dresden wurden die gleichen Tragödien und Komödien gespielt, die die Grundlage des Repertoires am höfischen Theater von Ludwig XV. in Fontainebleau und in der Comédie Française in Paris bildeten und in den Theatern Europas bis zum Ende des Jahrhunderts fortbestanden. Einige dieser Titel, die auf den Bühnen von August II. gespielt worden sind, kann man noch im Theater von Stanisław August Poniatowski finden.

Auch auf dem Boden des italienischen Theaters ist bei August II. die Neigung zu leichteren Gattungen zu sehen, zur komischen Oper, Drammi pastorali, Intermezzi und Komödie dell'arte. Unter ihnen dominierten mit Sicherheit die Werke mit der Musik von Giovanni Albert Ristori. Gerade die aus der polnischen Kasse finanzierte Künstlergruppe Comici italiani unter Tommaso Ristori brach Ende 1730 mit dem gleichen Repertoire, das vorher in Warschau und Dresden präsentiert worden war, von Warschau aus zur Eroberung Moskaus auf und wurde damit zu einer der ersten musikalischen Brücken zwischen Westeuropa und Russland.

Dank der engen Bindungen zwischen Warschau und Dresden, ebenfalls im Bereich der Kammermusik (sowohl der vokal-instrumentalen als auch der instrumentalen) war das Angebot des polnischen Hofes von August II. - wie es scheint - bedeutend reichhaltiger als in vielen anderen hervorragenden europäischen Zentren. Jedoch gaben sich die Italiener damals ausschließlich mit der eigenen Produktion zufrieden und die Franzosen, für die die italienische Musik lediglich in gewissen Zeiträumen die Quelle einer Inspiration darstellte, mussten auf die Entdeckung deutscher Komponisten sogar das halbe 18. Jahrhundert lang warten. In Warschau jedoch konzentrierten sich dagegen diese Leistungen sowohl der Franzosen als auch die der Italiener und Deutschen. Es wurden italienische und französische Kantaten und Arien, französische Ouvertures und italienische Sinfonien, Konzerte und Sonaten aufgeführt, darunter auch Kompositionen von Musikern des polnisch-sächsischen Hofes, ja selbst 
von Mitgliedern der polnischen Kapelle-J. J. Quantz, F. Benda, Heinrich Schultz, Johann Nicolaus Friese, Balthasar Villicus, Johann Blume. Selbstverständlich mussten sich im Warschauer Repertoire auch Instrumentalwerke der führenden Dresdner Komponisten befinden, wie zum Beispiel von Jan Dismas Zelenka, Johann David Heinichen oder Johann Georg Pisendel; ebenso Werke italienischer und französischer Meister, zum Beispiel von den in ganz Europa enorm populären Arcangelo Corelli und François Couperin. Mit Sicherheit waren auch zahlreiche Werke von Antonio Vivaldi und Georg Philipp Telemann aufgeführt worden, deren Werke - seinerzeit von Mitgliedern der polnischen Kapelle (J. J. Quantz, Johann Wolfgang Schmidt) kopiert - in den Sammlungen der Sächsischen Landes- und Universitätsbibliothek so zahlreich aufbewahrt werden. Ohne Zweifel stellten jedoch die Tänze, darunter die polnischen, den größten Teil des musikalischen Repertoires am Hofe von August II. Und paradoxerweise sind gerade diese Aufzeichnungen vollständig verschollen. In Warschau wurde selbstverständlich genauso getanzt wie an anderen europäischen Höfen, und zwar vor allem Menuetts, Kontredanse sowie Allemandes, jedoch war mit besonderem Eifer auch nach dem Rhythmus von Polonaisen, Mazurken und der russischen Kosaken getanzt worden.

Damit gehörte also - wie sich hieran zeigt - nicht nur der Dresdner, sondern auch der polnische Hof von August II. zu den interessantesten Musikzentren Mitteleuropas. Mehr noch, in dieser Zeit hatte Warschau gegenüber Dresden auch die bahnbrechende Initiative beim Ballet-d'action, direkt von Paris übernommen, oder aus Venedig - beim Intermezzi, oder - so wie es scheint - bei der in Sachsen überhaupt nicht existierenden Tragédie en musique. Zur Zeit der Herrschaft von August II. gab es im Bereich des Musiktheaters zwei miteinander konkurrierende Mächte - Frankreich und Italien. August II. sprach sich deutlich für Frankreich aus, verzichtete dabei jedoch nicht ganz auf das Schaffen der Italiener. Auf eine solche Auswahl hatten mit Sicherheit nicht nur seine intellektuelle und ästhetischen Neigungen ihren Einfluss, sondern auch seine Erfahrungen bei der grand tour durch Europa (1687-89) ${ }^{11}$ in der Jugendzeit.

August III. behielt bei der Organisation des musikalischen und des Theaterpersonals die von seinem Vater geschliffenen Muster bei und somit war das aus der polnischen Kasse bezahlte und in Warschau residierende Hauptensemble die sogenannte polnische Kapelle ${ }^{12}$. Ihre Besetzung stieg von über 10 Personen in den 30er Jahren bis auf ca. 30 Mitglieder in den 60er Jahren an und integrierte in immer größerem Maße polnische Künstler, unter anderem den Kastraten Stefan Jaroszewicz, den Oboisten Dominik Jeziomski sowie den Organisten Józef

${ }^{11}$ Siehe Alina Żórawska-Witkowska, Kawalerska tura Augusta II w świetle doświadczeñ muzyczno-teatralnych 1687-1689 [Die Tour des Junggesellen August II. im Lichte der musikalischen und Theatererfahrungen16871689], in: Arx Felicitatis. Księga ku czci profesora Andrzeja Rottermunda [ Arx felicitatis. Festschrift für Professor Andrzej Rottermund], Towarzystwo Opieki nad Zabytkami, Warszawa 2002, S. 625-635.

${ }^{12}$ Die Autorin bereitet augenblicklich eine Monografie vor, die der Musik am Warschauer Hof von August III. gewidmet ist und auf Quellen aus dem Sächsischen Hauptstaatsarchiv Dresden sowie aus der Sächsischen Landes- und Universitätsbibliothek beruht. 
MUZIKOLOŠKI ZBORNIK • MUSICOLOGICAL ANNUAL XL

Czanczik. Aus der polnischen Kasse wurde ebenfalls das Ensemble Comici italiani bezahlt, wenngleich der Hauptort ihres Aufenthaltes in Dresden lag. Tänzer, Sänger und einzelne Musikvirtuosen, die nach Warschau berufen wurden, rekrutierten sich jedoch aus den stricte Dresdner Ensembles. Am Warschauer Hof von August III. traten auch Musiker von Kapellen polnischer und litauischer Magnaten auf - Musiker des Hetmans Jan Klemens Branicki, des Podolsker Woiwoden Wacław Rzewuski, des Woiwoden von Kiew Franciszek Salezy Potocki sowie des Fürsten Hieronim Florian Radziwiłł. Das musikalische Angebot des königlichen Hofes wurde durch zahlreiche Konzerte ergänzt, die vom Ersten Minister Heinrich von Brühl in dessen Warschauer Palais sowie in der vorstädtischen Sommerresidenz in Młociny organisiert wurden. Brühl unterhielt eine eigene Kapelle, die sich hauptsächlich aus deutschen Musikern zusammensetzte, darunter die Kapellmeister Gottlieb Harrer, George Gebel und der Italiener Mattia Gherardi (später der erste Kapellmeister bei Stanisław August Poniatowski), der Cembalist Johann Gottlieb Goldberg (dank J. S. Bach ist er in der Geschichte der Musik verewigt) sowie die Lautenspieler - der Ukrainer Timofei Bielogradsky und der Deutsche Johann Kropfgans.

Ganz anders stellte sich die Situation während des siebenjährigen Krieges dar, als der ungewöhnlich längere Aufenthalt des Königs in Warschau eine Stabilisierung und ein Aufblühen des Musiklebens brachte. Zu dieser Zeit wurden neue Künstler engagiert, unter anderem die Sänger Castelli alias Caselli, Giuseppe Gallieni, Antonio Mariottini, Elisabeth Teyber alias Teuber und Luca (?) Fabris sowie die Tänzer Anetta Tagliavini-Lensi und Domenico Lensi; allmählich reisten auch Künstler nach Warschau, die nach dem Ausbruch dieses Krieges über Europa verstreut waren, wie zum Beispiel der Kapellmeister Johann Adolf Hasse (wahrscheinlich 1759 und 1760 in Warschau, mit Sicherheit dagegen in den Jahren 1762-63), die Sänger Bartolomeo Putini, Giuseppe Perini, Caterina Pilaja, Angelo Amorevoli und die Tänzerin Catherine Saint-George André.

Die prestigereichste Gattung am Hofe August III. war zweifelsohne das Dramma per musica ${ }^{13}$. Sie lernten die Polen sozusagen "von hinten" kennen, das heißt, dass sie zuerst zwei Parodien dieser Gattung sahen, die vom Ensemble Comici italiani aufgeführt wurden, unter der Leitung von Andrea und Antonio Bertoldi. Das waren: das 1739 aufgeführte Dramma ridicolo per musica Il Costantino mit der Musik von Giovanni Verocai (eines Schülers von Vivaldi), der im Juni 1738 seinen Dienst am Zarenhof in Petersburg beendete (dort arbeitete er bei der in Russland ersten Aufführung der Opera seria mit Francesco Araia zusammen) und auf seiner Rückreise nach Braunschweig in Warschau Station machte; sowie das 1748 aufgeführte Dramma per musica Le Contese di Mestre e Malghera per il trono, also eine nur leicht für die Warschauer Bedin-

\footnotetext{
${ }^{13}$ Siehe A. Żórawska-Witkowska, I drammi per musica di Johann Adolf Hasse rappresentati a Varsavia negli anni 1754-1763. Materialien der Warschauer Konferenz vom 10.-12. Dezember 1993, hrsg. von Irena Poniatowska und Alina Żórawska-Witkowska, Instytut Muzykologii Uniwersytetu Warszawskiego, Warszawa 1995, S. 123-148.
} 
gungen adaptierte venezianische Metamorfosi odiamorose mit dem Libretto von Anotnio Gori und der Musik von Salvatore Apolloni. In beiden Fällen handelte es sich um die Mitte der 20er Jahre des 18. Jh. in Venedig geschaffene satirische Oper, die die Reaktion des Theaters Comici auf den Pomp der Opera seria war, vor allem für das venezianische Teatro San Samuele ein ganz charakteristischer Typ, aber auch in London (1727) existierend und - wie man sieht ebenfalls in Warschau.

Die reine Gattung des Dramma per musica und dies in der damals herausragendsten Gestalt, das von den größten Meistern Pietro Metastasio und Johann Adolf Hasse geschaffen wurde, konnte Warschau erst im Herbst 1754 kennen lernen (L'eroe cinese). Heimisch wurde diese Gattung im Warschauer Opernhaus erst während des Krieges, als man 10 andere Werke von Hasse auf die Bühne brachte, von denen in 9 Fällen das Libretto von Metastasio stammte und eins vom Librettisten Giovanni Claudio Pasquini (Arminio, 1761). Dank dieser 124 Aufführungen an der Zahl, die in den Jahren 1754 und 1759-63 im königlichen Opernhaus gegeben worden sind, bekamen die polnischen und litauischen Zuschauer eine solide Dosis paneuropäischer Kunst und diese in der bestmöglichen Ausgabe. Diese Opern habe ich in 3 Gruppen eingeteilt:

1. Die neusten Leistungen Hasses, die während des Krieges in Venedig, Neapel oder in Wien entstanden - Nitteti, Artaserse, Zenobia ${ }^{14}$, Il trionfo di Clelia ${ }^{15}$;

2. Werke, die in Dresden, kurz vor dem Ausbruch des Krieges, komponiert wurden - Olimpiade, Il re pastore, Arminio;

3. Wiederaufnahmen von älteren Werken - Demofoonte, Ciro riconosciuto, Semiramide riconosciuta.

Die Partituren der Werke, die zu den beiden ersten Gruppe gehören, tragen Spuren von eigenhändigen und nicht selten weitgehenden Eingriffen des Komponisten in seinen ursprünglichen Musiktext, die von seiner direkten Begeisterung bei der Vorbereitung der Warschauer Premieren zeugen. Wohingegen die Partituren der dritten Gruppe, von Kopisten angefertigt, keinerlei solche Eingriffe aufweisen und wo sich der musikalische Inhalt von den früheren Versionen nicht unterscheidet und nur auf minimale Weise den Warschauer Aufführungsbedingungen angepasst worden waren.

Das Ensemble Comici italiani, unter dessen Mitglieder solche Berühmtheiten des venezianischen Theaters waren wie Giovanna Casanova, Marta Focari, genannt La Bastona, Francesco Golinetti, Giovanni Camillo Canzachi sowie Cesare D'Arbes, gab im Verlaufe von drei Warschauer Spielzeiten (1738-39, 1748-49, 1754) rund 60 Aufführungen, bei denen sie nicht nur die erwähnten

\footnotetext{
${ }^{14}$ Siehe: Alina Żórawska-Witkowska, The Good Wife Zenobia, in: 8th Baroque Opera Festiwal 14. 11. 2000 - 18. 01. 2001, Warsaw 2000, Warsaw Chamber Opera, S. 428-432; Reinhard Strohm, Zenobia: voices and authorship in opera seria, in: Johann Adolf Hasse in seiner Epoche und in der Gegenwart. Studien zur Stil- und Quellenproblematik, hrsg. von Szymon Paczkowski und Alina Żórawska-Witkowska, Instytut Muzykologii Uniwersytetu Warszawskiego, Warszawa 2002, S. 53 - 81.

${ }^{15}$ Siehe A. Żórawska-Witkowska, 'Il Trionfo di Clelia' di Johann Adolf Hasse: versione varsaviana, in: ebenda S. $83-98$.
} 
satirischen Opern, sondern auch Komödien dell'arte und literarische Komödien von Carl Goldoni spielten, die jener Meister nicht selten mit dem Gedanken an die schauspielerischen Fähigkeiten der genannten Künstler geschrieben hatte. Canzachi bewies auch ein eigenes dramaturgisches Talent. So wurden die Warschauer Vorstellungen der Comici italiani von je 3 Balletten verziert. Am häufigsten waren es vom Genre her Divertissements, aber 1754 tauchten auch schon Ballets d'action auf. Die »französische« Musik zu diesen Balletten komponierte Johann Adam, der Bratschist der Dresdner Kapelle.

Hinzugefügt muss auch werden, dass im Theaterrepertoire des Warschauer Hofes ebenfalls Pantomimen enthalten waren - unter anderem die zweimal aufgeführte Pantomime Der von Pantalon verfolgte Arlequin (1759 und 1760), die von der deutschen Truppe Martin Lepperts dargeboten worden war, sowie französische Komödien - im königlichen Opernhaus wurden sie 1762 vom kommerziellen Ensemble der französischen Komödie unter der Leitung eines gewissen J.F. Albani gespielt (es waren Werke der führenden französischen Dramaturgen wie: Jean-François Regnard, Pierre Marivaux, François-Marie Volter, Marc-Antoine Le Grand, Pierre-Claude de La Chaussé, Jean La Fontaine, Florent Carton Dancourt, Philippe Nericault Destouches, Louis Bossy, Denis Diderot und Moličre). Darüber hinaus waren in der Stadt an den Lehranstalten der Jesuiten, der Piaristen sowie der Teatriner Schultheater aktiv. Die dort von Schülern jener Lehranstalten aufgeführten Dramen in Lateinisch, Französisch, Italienisch und Polnisch wurden oft mit einem musikalischen Rahmen versehen.

Was die Kirchenmusik anbelangt, die bekanntlich mit einem engagierten Pietismus am Dresdner Hof von August III. gepflegt wurde, sind wir in der Lage, und dies trotz zahlreicher Aufzeichnungen, die die Teilnahme der königlichen Sänger und Instrumentalisten nicht nur in der Hofkirche, sondern auch in den in verschiedensten Warschauer Gotteshäusern zelebrierten Gottesdiensten bestätigen (bei den Augustinern, Dominikanern, Jesuiten, Piaristen, Reformatoren, Visitantinnen, Eucharistinerinnen), lediglich in sehr wenigen Fällen die konkret aufgeführten Werke zu nennen. Dies sind vor allem mindestens 4 Oratorien von Hasse, einige von ihnen wurden ohnedies mehrmals wiederholt, Il Cantico de' tre fanciulli, I pellegrini al Sepolcro di Nostro Signore, Sant'Elena al Calvario, La Conversione di Sant'Agostino, sowie verschiedene andere Werke - Messen, Psalme, Motetten, Stabat Mater, die von den Künstlern komponiert worden waren, die in Verbindung mit dem königlichen Hofe standen - Giovanni Alberto Ristori, Johann Michael Breunich, Niccolň Porpora, Johann Georg Schürer, Tobias Buz, Gottlieb Harrer vielleicht sogar Johann Sebastian Bach. Im Falle des zuletzt genannten Meisters geht es um die Aufführung vom 27. Februar 1763 in der evangelischen Kapelle aus Anlass des Friedensschlusses in Hubertsburg, bei der wahrscheinlich die zwei Chorkantaten Sey Lob und Ehr dem höchsten Gut (BWV 117) sowie Herr Gott, dich loben wir (BWV 130) erklangen.

Das vokal-instrumentale Konzertrepertoire bildeten zu jener Zeit vor allem italienische gelegentliche Serenate, die bei Festen am Hofe gespielt und mit denen Geburts- und Namenstage der königlichen Familie gewürdigt wurden. 
Autoren von 11 solcher uns heute bekannten Werke sind die Dichter des polnisch-sächsischen Hofes - Stefano Benedetto Pallavicini, Giovanni Claudio Pasquini, Giovanni Ambroggio Migliavacca sowie der unerreichbare poeta cesareo Pietro Metastasio; die Musik komponierten Ristori, Breunich und Gherardi. Es gab ebenfalls die von den Darstellern (Sängern) aus verschiedenen italienischen Arien zusammengesetzten Werke, die sogenannte Pasticcia, z.B. mit der Musik von David Peres und Leonardo Leo.

Um die damals in Warschau gespielten Instrumentalwerke einzugrenzen, müssen wir deren Komponisten erneut innerhalb des Kreises von Musikern der Dresdner sowie der polnischen und der Brühlschen Kapelle suchen. Sie haben wohl alle damaligen Gattungen gepflegt, wie zum Beispiel: Sinfonien, Divertimenti und Partite (Ouvertures), Solokonzerte und Concerti grossi, Cembalo- und Trio-Sonaten, verschiedene Quadri und Duette, Solowerke für Violine, Violoncello, Flöte, Oboe, Cembalo, Laute und andere Instrumente. Solche Werke besaßen in ihrem Schaffen zum Beispiel der Geiger Johann Georg Pisendel, der Flötist Pietro Grassi Florio (der spätere Solist der Pariser Concert Spirituel und Londoner Bach-Abel Concerts), der Geiger und Spaßmacher Pietro Mira, genannt Pedrillo (früher am Zarenhof von Anna Iwanowna in Petersburg tätig), der Cembalist Johann Gottlieb Goldberg und der Lautenspieler Johann Kropfgans. Vielleicht präsentierten auch die hochwohlgeborenen Amateure irgendeine eigene Komposition, wie zum Beispiel der Königssohn Karl - ein Flötist, Graf Wielhorski - ein Lautenspieler sowie der Warschauer Landrat Alojzy Fryderyk von Brühl - ein Perkussionist und Cembalist, seine Schwester, die Frau Hofmarschall Amalia Mniszchowa - eine Sängerin, oder der litauische Großhetman Michał Kazimierz Ogiński - ein Geiger, Klarinettist, Harfenist und Komponist. In den 50er und 60er Jahren fanden die Konzerte schließlich auch nicht mehr nur beim König und seinem Ersten Minister statt, sondern ebenso in den Residenzen der verschiedenen weltlichen und geistlichen Magnaten, wie zum Beispiel beim Hofmarschall Jerzy August Mniszech oder beim Krakauer Bischof Kajetan Sołtys.

Somit war das in Warschau oft von den auf ihrem Gebiet besten Künstlern dargebotene Repertoire während der Herrschaft von August III. von einer enormen Verschiedenartigkeit der Gattungen und Stile geprägt. Es überwog der italienische Stil (die italienische Musik lernte August II. lieben während seiner Aufenthalte in Italien ${ }^{16}$ ), aber es grenzte sich bereits deutlich dieser deutsche "vermischte Geschmack"ab. Des weiteren könnten die Zechen der Kaufleute und die kirchlichen Bruderschaften ein Hort für das nationale Element gewesen sein. Damals konkurrierten in Warschau zwei solche Bruderschaften miteinander: die so genannten Serben (polnisch Serbaki - sie wurde 1717 in der Karmeliterkirche gegründet), die hauptsächlich während der bürgerlichen

${ }^{16}$ Alina Żórawska-Witkowska. Esperienze musicali del principe polacco Federico Augusto in viaggio attraverso l’Europa (1711-1719), "Studi musicali« XX., 1991, Nr. 1, S. 155-173. 
Familienfeiern beschäftigt wurden, sowie die besser als sie musikalisch ausgebildeten »Mariacki«-Musiker (in der Kirche der Heiligen Jungfrau Maria bestätigt), die bei Feierlichkeiten in den Warschauer Gotteshäusern aufwarteten.

Die Werke, die am königlichen Hofe aufgeführt worden sind, waren generell im Stil galant gehalten, daher existierten die barocken Partiten und die bereits in ihrer Konzeption klassischen Sinfonien, Concerti grossi und Solokonzerte, Triosonaten sowie Prototypen des Streichquartetts; Divertissements und Ballets d'action; die Komödie dell'arte sowie die literarische Komödie nebeneinander. Die Mehrheit der Werke bedeutete ohne jeden Zweifel eine Replik des Dresdner Repertoires, doch es kamen in Warschau auch originale Leistungen vor, die dem damaligen Dresden unbekannt waren - die italienische satirische Oper, das französische Ballet d'action, die Janitscharenmusik, sowie z.B. die Auftritte von Kosaken. Es gab aber desgleichen Werke, die speziell für Warschau komponiert worden waren, zum Beispiel Gelegenheitsserenate sowie Dramma per musica Zenobia. Und wenngleich nun das damalige Warschau de facto eine musikalische Expositur Dresdens war, so jedoch eine Expositur, die gewisse individuelle Züge besessen hatte.

Trotz der ganzen Herrlichkeit der Warschauer Musikkultur in der so genannten Sachsenepoche muss jedoch ganz deutlich hervorgehoben werden, dass Warschau damals im Bereich des professionellen Schaffens der fast ausschließlich nehmende Teil gewesen war; gleichwohl schöpfte die Stadt aus den zu jener Zeit besten Quellen, die Paris, Venedig und Dresden hießen. Die Kirchenmusik (die katholische), das einzige Gebiet, auf dem polnische Komponisten die reichhaltige nationale Tradition des 17. Jh. nach der Übernahme des Throns durch August II. hätten fortsetzen können, hatte nicht die Unterstützung dieses Monarchen gefunden und somit erlosch dann auch jene Tradition.

Beide Sachsenkönige trugen zweifelsohne zum Verfall des örtlichen kompositorischen Schaffens bei; dabei führten sie Warschau aber in den Kreis der westeuropäischen Kultur ein. Sie trugen damit zur Europäisierung der Musikkultur dieser Stadt bei und hatten Anteil - wenn nicht an der gesamteuropäischen, dann zumindest an der deutschen Karriere der Polonaise, die der Grund dafür war, dass sich in der damaligen Musiktheorie der Begriff vom polnischen Stil herausbildete $^{17}$. Und zweifelsohne wurde ebenfalls der Appetit der Bevölkerung auf Musik und Theater geweckt und zwar so, dass während der Herrschaft von Stanisław August Poniatowski (1764-1795) eine regelrechte Explosion auf dem Gebiet des öffentlichen, demokratischen Theaters stattfand, als es im Jahre 1765, also nur 2 Jahre nach dem Tod von August III., zu funktionieren begann. Zu Zeiten Stanisław August Poniatowskis wurden in der Hauptstadt bereits insgesamt mindestens 245 italienische, französische, deutsche und polnische Opern auf-

\footnotetext{
${ }^{17}$ Johann Mattheson, Kern melodischer Wissenschaft, Hamburg 1737, Reprint 1954, S. 116-117; Johann Adolf Scheibe, Der critische Musicus, (15. Stück, 17. Sept. 1737), Leipzig 1745, Reprint 1970, S. 145.Siehe auch Memorial Johann Sebastian Bachs vom 23.08.1730 an die Verwaltungsbehörden Leipzigs, zit. in: Philipp Spitta, Johann Sebastian Bach, ..., Leipzig 1880, Bd. II, S. 78.
} 
geführt sowie mindestens 206 Ballette. Vor über einem Jahrzehnt ${ }^{18}$ hatte ich bereits die Gelegenheit hier in Ljubljana darüber zu sprechen.

Aus polnischer Sicht ist das wichtigste Ergebnis der Poniatowski-Epoche jedoch die Wiedergeburt des lokalen kompositorischen Milieus und die Entstehung der ersten nationalen Opern, die zum Grundrepertoire für andere Theater der Republik Beider Nationen gehörten und dieser Gattung im 19. Jahrhundert die Entwicklungsrichtung vorgaben.

War die hier vorgestellte musikalische Situation Warschaus damals für die Länder »Mitteleuropas« typisch oder auch spezifisch? Mit Sicherheit sowohl ein bisschen typisch als auch ein bisschen spezifisch.

In der Hoffnung, dass es unsere Konferenz erlaubt, die Bindungen zu erkennen, die die verschiedenen Länder in diesem Teil unseres Kontinents verbanden, und die Antworten hierauf sowie auf ähnliche Fragen erleichtert, möchte ich den Organisatoren sehr herzlich danken für die Einladung zur Teilnahme an dieser Konferenz, vor allem auch deshalb, weil Polens Zugehörigkeit zu jener mitteleuropäischen Familie für sie nicht gerade so selbstverständlich gewesen war.

${ }^{18}$ Alina Żórawska-Witkowska. La Varsavia dei tempi di Stanislao Augusto (1764-1795) - uno dei centra europei della vita artistica, in: Evropski glasbeni klasicizem in njegov odmev na Slovenskem, Mednarodni simpozij Ljubljana 26.-28.10.1988, Red. Dragotin Cvetko, Danilo Pokorn, Slovenska akademija znanosti in umetnosti, Ljubljana 1988, S. 149-159. 